were daily, weekly, monthly, every 3 months or never. Multivariate logistic regression on weighted data was performed to assess variation by sex, age group (50 to state pension age (SPA), SPA to 74, 75 and over), and wealth quintile.

Results 5,142 core participants responded to both W9 and CW1. Of these, 553 (10.75\%; 95\% confidence interval (CI) 9.71 to 11.89 ) reported never using the internet in W9 and $733(14.26 \% ; 13.05$ to 15.57$)$ in CW1. Of those aged 75 and older, $320(30.64 \% ; 26.87$ to 34.87$)$ were 'never users' in W9 and $419(40.03 \%$; 35.51 to 45.03$)$ in CW1. Univariate analysis found that the odds of reporting 'never use' were higher for women than men (W9 odds ratio (OR) 1.39; (CI) 1.11 to 1.73 , CW1 $1.35 ; 1.11$ to 1.66$)$, older age groups (W9 $4.21 ; 3.36$ to 5.27 , CW1 $4.24 ; 3.50$ to 5.14 ), and less wealthy quintiles (W9 $1.18 ; 1.10$ to 1.26 , CW1 $1.19 ; 1.11$ to 1.27). Multivariate analysis found that age was the most important predictor of never using the internet. The odds for older age groups were 4.73 ; 3.81 to 5.89 (W9) and 4.93; 4.09 to 5.93 (CW1). The differences between women and men, and between wealth quintiles, were no longer statistically significant.

Conclusion The proportion of participants reporting that they never used the internet increased slightly during the pandemic and included 4 in every 10 of those aged 75 and older. A limitation is that W9 data were collected using a paper survey delivered by an interviewer, and CW1 were administered over the phone or internet. Overall, there is a substantial risk that a 'digital first primary care' policy will create barriers for those aged over 75 years. It will be important to maintain alternative access routes to avoid increasing barriers to health care access and subsequent inequalities in the care provided to older people in England.

\section{OP34 ETHNIC AND EDUCATIONAL INEQUALITIES IN COVID-19 VACCINE HESITANCY: CROSS-SECTIONAL ANALYSIS OF THE UK HOUSEHOLD LONGITUDINAL STUDY}

${ }^{1}$ Elaine Robertson*, ${ }^{2}$ Kelly S Reeve, ${ }^{3}$ Claire L Niedzwiedz, ${ }^{2}$ Jamie Moore, ${ }^{4}$ Margaret Blake, ${ }^{1}$ Michael J Green, ${ }^{1,5}$ S Vittal Katikireddi, ${ }^{2}$ Michaela Benzeval. ${ }^{1}$ MRC/CSO Social and Public Health Sciences Unit, University of Glasgow, Glasgow, UK; ${ }^{2}$ Institute for Social and Economic Research, University of Essex, Colchester, UK; ${ }^{3}$ Institute of Health and Wellbeing, University of Glasgow, Glasgow, UK; ${ }^{4}$ Social Research Institute, Ipsos MORI UK Ltd, London, UK; ${ }^{5}$ Public Health Scotland, Public Health Scotland, Glasgow, UK

\subsection{6/jech-2021-SSMabstracts.34}

Background Vaccination is crucial to address the COVID-19 pandemic but inequalities in uptake may exacerbate existing health inequalities. We investigate the UK prevalence of COVID-19 vaccine hesitancy, identify which population subgroups are more likely to be vaccine hesitant, and report stated reasons for hesitancy.

Methods Nationally representative survey data from 12,035 participants were collected from 24th November to 1 st December 2020 for wave 6 of the UK Household Longitudinal Study ('Understanding Society') COVID-19 web survey. Participants self-reported ethnicity, highest educational attainment, gender, age, how likely they would be to have a vaccine if offered and their main reason for hesitancy. Weighted cross-sectional analysis assessed the prevalence of vaccine hesitancy and logistic regression models estimated independent associations.

Results Overall vaccine hesitancy was low (18\% unlikely/very unlikely). Vaccine hesitancy was higher in women $(21.0 \%$ vs
$14.7 \%$ in men), in younger age groups $(26.5 \%$ in $16-24$ year olds vs $4.5 \%$ in $75+$ ) and in those with lower education levels $(18.6 \%$ no qualifications vs $13.2 \%$ degree qualified). Vaccine hesitancy was high in Black (71.8\%) and Pakistani/ Bangladeshi (42.3\%) ethnic groups. Odds ratios for vaccine hesitancy after adjustment for age and gender were 13.42 (95\% CI:6.86, 26.24) in Black, 2.54 (95\% CI:1.19, 5.44) in Pakistani/Bangladeshi groups, and 1.76 (95\% CI:1.10, 2.82) for Other White (including Eastern European) ethnic groups (compared to White British/Irish). Vaccine hesitancy was not higher in all minority ethnic groups; for example, ORs were 1.11 (95\% CI:0.64, 1.95) for Indian ethnicity and 0.67 (95\% CI:0.24, 1.87) for Other Asian (including Chinese) ethnicity. Lower education was also related to vaccine hesitancy (no qualifications versus degree OR 3.54; 95\% CI:2.06, 6.09) but ethnic differences largely remained when education was included in the model. For those who were vaccine hesitant the most common stated reason for hesitancy was concerns over unknown future effects (42.7\%). However, when compared to the White British/Irish group, Black participants were more likely to state they 'Don't trust vaccines' $(29.2 \%$ vs $5.7 \%$ ) and the Pakistani/Bangladeshi ethnic group more frequently cited worries about side-effects (35.4\% vs $8.6 \%)$.

Conclusion Vaccine hesitancy is strongly associated with education and ethnicity, with marked ethnic heterogeneity. Black and Pakistani/Bangladeshi participants reported considerably greater vaccine hesitancy than White British/Irish ethnicity, but some minority ethnic groups did not. Educational inequalities did not account for ethnic differences. Vaccine programmes need to understand reasons for vaccine hesitancy within specific population sub-groups and take urgent action to improve uptake.

\section{OP35 RACE, ETHNICITY AND COVID-19 VACCINATION: A QUALITATIVE STUDY OF UK HEALTHCARE STAFF}

${ }^{1}$ Charlotte Woodhead*, ${ }^{2}$ Juliana Onwumere, ${ }^{1}$ Rebecca Rhead, ${ }^{3}$ Monalisa Bora-White, ${ }^{1}$ Zoe Chui, ${ }^{4}$ Naomi Clifford, ${ }^{1}$ Cerisse Gunasinghe, ${ }^{1}$ Hannah Harwood, ${ }^{5}$ Paula Meriez, ${ }^{6}$ Ghazala Mir. ${ }^{1}$ Psychological Medicine, Kings College London, London, UK; ${ }^{2}$ Psychology, Kings College London, London, UK; ${ }^{3}$ Avon and Wiltshire Mental Health NHS Partnership Trust, Bath, UK; ${ }^{4}$ Nottinghamshire Healthcare NHS Foundation Trust, Nottingham, UK; ${ }^{5}$ Cambridgeshire and Peterborough NHS Foundation Trust, Cambridge, UK; ${ }^{6}$ Leeds Institute of Health Sciences, University of Leeds, Leeds, UKi ${ }^{7}$ Psychology, City University, London, UKi ${ }^{8}$ Adult Nursing, Kings College London, London, UK; ${ }^{9}$ London School of Hygiene and Tropical Medicine, London, UK

\subsection{6/jech-2021-SSMabstracts.35}

Background COVID-19-related inequities experienced by racial and ethnic minority groups including healthcare professionals mirror wider health inequities, which risk being perpetuated by lower uptake of vaccination. We aim to better understand lower uptake among racial and ethnic minority staff groups to inform initiatives to enhance uptake.

Methods Twenty-five semi-structured interviews were conducted (October 2020-January 2021) with UK-based healthcare staff. Data were inductively and thematically analysed.

Results Vaccine decision-making processes were underpinned by an overarching theme, 'weighing up risks of harm against potential benefits to self and others'. Sub-themes included 'fear of harm', 'moral/ethical objections', 'potential benefits to self and others', 'information and misinformation', and 'institutional or workplace pressure'. We identified ways in which these were weighted more heavily towards vaccine hesitancy 
for racial and ethnic minority staff groups influenced by perceptions about institutional and structural discrimination. This included suspicions and fear around institutional pressure to be vaccinated, racial injustices in vaccine development and testing, religious or ethical concerns, and legitimacy and accessibility of vaccine messaging and communication.

Conclusion Drawing on a critical race perspective, we conclude that acknowledging historical and contemporary abuses of power is essential to avoid perpetuating and aggravating mistrust by de-contexualising hesitancy from the social processes affecting hesitancy, undermining efforts to increase vaccine uptake.

\section{OP36 IS RISK OF BIAS UNDERESTIMATED IN POORLY CONDUCTED SYSTEMATIC REVIEWS USING COCHRANE'S RISK OF BIAS TOOL FOR NON- RANDOMISED STUDIES? A META-EPIDEMIOLOGICAL REVIEW}

Erik Igelström*, Mhairi Campbell, Peter Craig, Srinivasa Vittal Katikireddi. MRC/CSO Social and Public Health Sciences Unit, University of Glasgow, Glasgow, UK

\subsection{6/jech-2021-SSMabstracts.36}

Background Systematic reviews of evidence frequently help inform research, clinical guidelines, and policy. Although randomised controlled trials (RCTs) are ideal, many areas of public health rely on evidence from non-randomised studies which are more susceptible to bias. 'Risk Of Bias In Non-Randomised Studies of Interventions' (ROBINS-I) is a widely used critical appraisal tool developed by Cochrane in 2016, which assesses risk of bias on an absolute scale, such that a low riskof-bias study is equivalent to a well-conducted RCT. ROBINSI has been seen as a major methodological innovation, but its complexity has led to concerns that it may be misapplied. We review for the first time how ROBINS-I is used in a sample of recent systematic reviews.

Methods Systematic reviews using ROBINS-I were identified by forward citation and keyword/abstract searches in six databases, restricted to January and February 2020. The review protocol was preregistered in PROSPERO (CRD42020170785). Reported ROBINS-I ratings and data on how ROBINS-I was used were extracted from each review. Methodological quality of reviews was assessed using AMSTAR 2 ('A MeaSurement Tool to Assess systematic Reviews'). Mixed-effects partial proportional odds regression was used to assess associations between review characteristics (e.g. methodological quality and industry funding) and risk-ofbias ratings. Screening and quality appraisals were conducted independently by two reviewers.

Results Of 181 hits, 124 reviews were analysed with data extracted on 1,344 included studies. Risk of bias was reported as serious/critical for $54.8 \%$ of included studies, most commonly due to confounding, but $8.0 \%$ reported low risk of bias. Poorly conducted reviews were more likely to report lower risk-of-bias ratings, with an apparent dose-response relationship. Compared to reviews with moderate/high AMSTAR 2 rating, odds of low risk-of-bias ratings were higher in lowquality reviews (odds ratio: 1.89 [95\% confidence interval: $0.36-9.94]$ ), and considerably higher in critically low-quality reviews (4.70 [1.01-21.78]). Competing interests and industry funding were not uniformly predictive of higher or lower ratings, although these analyses had low statistical power.
Deviations from the guidance of the tool were seen in $40.3 \%$ of studies, with $20.2 \%$ reporting ratings using a non-standard scale.

Discussion Systematic reviews conducted using Cochrane's recommended tool for non-randomised studies may misleadingly suggest a robust evidence base exists when used by reviewers without adequate epidemiological expertise. This may lead to misleading conclusions, especially for public health guidelines. Greater training and expertise are required to ensure that widespread use of the tool does not lead to an increase in misleading reviews.

\section{Friday 17 September}

\section{Session: Food \& Food Policy, $09.00-11.30$}

\section{OP37 CHILDHOOD CONSUMPTION OF ULTRA-PROCESSED FOODS AND LONG-TERM ADIPOSITY TRAJECTORIES: FINDINGS FROM A UK BIRTH COHORT STUDY}

${ }^{1}$ Kiara Chang*, 2,3,4 Neha Khandpur, ${ }^{2,3}$ Daniela Neri, ${ }^{5}$ Mathilde Touvier, ${ }^{6}$ Inge Huybrechts, ${ }^{1}$ Christopher Millett, ${ }^{1}$ Eszter Vamos. ${ }^{1}$ Public Health Policy Evaluation Unit, Imperial College London, London, UK; ${ }^{2}$ Department of Nutrition, University of Sao Paulo, Sao Paulo, Brazil; ${ }^{3}$ Center for Epidemiological Research in Nutrition and Health, University of Sao Paulo, Sao Paulo, Brazil, ${ }^{4}$ Department of Nutrition, Harvard T. H. Chan School of Public Health, Boston, US; ${ }^{5}$ INSERM U1153, Sorbonne Paris Nord University, Bobigny, France; ${ }^{6}$ Nutrition and Metabolism Branch, International Agency for Research on Cancer, Lyon, France

\subsection{6/jech-2021-SSMabstracts.37}

Background Worldwide consumption of ultra-processed foods is continued to rise. Growing evidence has linked ultra-processed food consumption with elevated risks of obesity, noncommunicable diseases, and mortality in adults. Associations between ultra-processed food consumption and long-term adiposity trajectories have never been investigated in children.

Methods Data were obtained from the Avon Longitudinal Study of Parents and Children (ALSPAC), a prospective birth cohort study conducted in Avon County, south-west England. Participating children with baseline dietary intakes collected using 3-day food diaries and repeated measures of adiposity outcome were included and followed up from ages 7 to 24 years (1998-2017). Adiposity outcomes included objectively assessed anthropometrics (body mass index, weight, waist circumference) and dual-energy X-ray absorptiometry measurements (fat and lean mass index, body fat percentage). All foods and drinks consumed were categorised according to the degree of processing applying the NOVA food classification system. Individual's consumption of ultra-processed foods was derived as a percentage of its weight contribution (gram per day) in the total diet and categorised into quintiles. Associations between quintiles of ultra-processed food consumption and trajectories of adiposity outcomes were evaluated using linear growth curve models and adjusted for study covariates.

Results A total of 9025 children $(49.6 \%$ female $)$ were followed up over a median (IQR) of 10.2 (5.2-16.4) years. Mean (SD) ultra-processed food consumption at baseline from the lowest to highest consumption quintiles was $23.2 \%$ (5.0\%), 34.7\% (2.5\%), 43.4\% (2.5\%), 52.7\% (2.8\%) and $67.8 \%(8.1 \%)$. Trajectories of body mass index, fat mass index, weight and waist circumference increased significantly 